\title{
ETNOBOTÁNICA, FENOLOGÍA Y PRODUCCIÓN DE VAINAS EN ÁRBOLES DE Cassia grandis L. f. DEL CENTRO DE CHIAPAS
}

\author{
ETHNOBOTANY, PHENOLOGY AND POD YIELD IN TREES OF \\ Cassia grandis L. f. AT CENTRAL CHIAPAS
}

\author{
Ma. Eugenia Velasco Zebadúa ${ }^{1 *}$, Raúl A. Perezgrovas Garza², Víctor A. González Hernández \\ Alfonso Hernández Garay ${ }^{3}$, Miguel Salvador Figueroa ${ }^{4}$ y Jaime Martínez Tinajero ${ }^{5}$
}

\begin{abstract}
${ }^{1}$ Facultad de Medicina Veterinaria y Zootecnia, Universidad Autónoma de Chiapas, Campus II. Rancho "San Francisco" Km 8, Camino Ejido "Emiliano Zapata" Apartado Postal 392. 29050, Tuxtla Gutiérrez, Chiapas, México. ${ }^{2}$ Instituto de Estudios Indígenas, Universidad Autónoma de Chiapas, Campus III. Eje 3 Lic. Javier López Moreno s/n. Barrio de Fátima. 29264, San Cristóbal de Las Casas, Chiapas, México. ${ }^{3}$ Postgrado de Recursos Genéticos y Productividad. Colegio de Posgraduados-Campus Montecillo. km 36.5 carretera México-Texcoco. 56230, Montecillo, Texcoco, Estado de México. ${ }^{4}$ Centro de Biociencias, Universidad Autónoma de Chiapas, Campus IV. Tapachula, Chiapas, México. ${ }^{5}$ Facultad de Ciencias Agrícolas, Universidad Autónoma de Chiapas, Campus IV. Entronque Carretera Costera y Estación Huehuetán. Huehuetán, Chiapas.
\end{abstract}

* Autor para correspondencia (mvelascoz@yahoo.com.mx)

\section{RESUMEN}

Dada la estacionalidad forrajera, la escasez y elevado costo de concentrados proteínicos para la alimentación animal en la región central de Chiapas, México, este trabajo se hizo para conocer la etnobotánica, las características edafoclimáticas donde crece, la morfometría, fenología y el rendimiento de vainas por árbol de cañadonga (Cassia grandis L.f.), y determinar un modelo que estime la producción por árbol. Entre 2007 y 2008 se aplicó una encuesta a productores de la región para conocer la etnobotánica asociada con esta arbórea. Debido a su escasez, se trabajó con nueve ejemplares encontrados en los municipios de Tuxtla Gutiérrez (TX), San Fernando (SF) y Tecpatán (TP). En ellos se midieron los cambios semanales en los componentes morfológicos, así como las dimensiones, número, peso individual y total de vainas por árbol, número de semillas por vaina y peso de 100 semillas. Solamente $1 \%$ de los encuestados conoce algún uso de las vainas. Los árboles crecen en pH del suelo $y$ altitudes diferentes a las reportadas en Centroamérica. La etapa de floración ocurre de febrero a mayo, y la maduración de la vaina dura 18 semanas. El número de vainas varía de 239 a 1226 por árbol y el rendimiento de 33 a $254 \mathrm{~kg}$ por árbol. $\mathrm{El}$ árbol con las vainas de mayor $(P \leq 0.05)$ longitud $y$ peso fue $\mathrm{TP}$ $(54.3 \mathrm{~cm}$ y $497.3 \mathrm{~g})$, y las vainas más anchas $(P \leq 0.05)$ fue en San José Terán (TE, $3.8 \mathrm{~cm})$. Se encontró relación lineal entre la altura del árbol y la producción de vainas $\left(r=0.91^{* *}\right)$.

Palabras clave: Cassia grandis, ciclo fenológico, producción de vainas.

\section{SUMMARY}

Given the seasonality of grass production, the low availability and high costs of commercial protein concentrates utilized in animal production in the central region of Chiapas, México, this study was carried out in order to learn about the ethno-botany of the cañadonga tree (Cassia grandis L.f.). Description of the soil and climatic characteristics where it grows, the morphometry, the phe- nologic cycle, pod yield per tree, and the design of a model to establish pod yield per tree are products of this study. Between 2007 and 2008, a survey was conducted among livestock producers from the region to gather the ethnobotany associated to this legume tree. Due to its scarcity, the study included nine trees found in the Tuxtla Gutiérrez (TX), San Fernando (SF) and Tecpatán (TP) regions. In these trees, weekly changes were observed regarding the morphologic components, along with their dimensions, pod number, individual pod weight and total pod weight per tree, as well as number of seeds per pod and weight of 100 seeds. Only $1 \%$ of the livestock producers knew an use for the pods. Trees grow at different soil $\mathrm{pH}$ and altitudinal level than those reported in Central America. Flowering period ocurrs from February to May, and pod maturation lasts 18 weeks. The amount of pods ranges from 239 to 1226 per tree, and pod yield from 33 to $254 \mathrm{~kg}$ per tree. The tree with the largest and heavier pots $(P \leq 0.05)$ was $T P(54.3 \mathrm{~cm}$ and $497.3 \mathrm{~g})$, while the widest pods $(P \leq \mathbf{0 . 0 5})$ were found in San José Terán $(\mathrm{TE}, 3.8 \mathrm{~cm})$. A linear relationship was found between tree height and pod yield $(r=0.91 * *)$.

Index words: Cassia grandis, phenologic cycle, pod production.

\section{INTRODUCCIÓN}

En el trópico de México la producción animal se basa principalmente en el pastoreo de gramíneas con metabolismo fotosintético $\mathrm{C}_{4}$, las cuales presentan marcada estacionalidad en la producción de forraje y limitaciones en su valor nutritivo, lo que sumado a un manejo inadecuado trae como consecuencia que la producción ganadera en pastoreo sea una cuarta parte de la obtenida en el trópico y sub-trópico de Florida en EE. UU. (Mena-Urbina et al., 2007). La introducción de gramíneas mejoradas en esta región ha favorecido la producción pecuaria, donde se produce $25 \%$ de la leche y 
$35 \%$ de la carne del total nacional, pero no ha sido suficiente para incrementar la productividad (Enríquez et al., 1999), porque la mayoría de los productores no enmienda la falta de proteína, ya sea por escasez del producto o por el alto costo económico de los concentrados comerciales disponibles en el mercado local, lo que acentúa la baja productividad, sobre todo durante la época seca.

Lo anterior ha motivado la evaluación de recursos alimenticios alternativos eficientes y viables desde el punto de vista económico; al respecto, en algunos países de Latinoamérica se han explorado plantas arbóreas y arbustivas, en su mayoría leguminosas, por sus elevados contenidos de proteína en hojas, vainas y semillas (18.3 a 20,10 a 20 y 35 a $45 \%$, respectivamente), alto valor nutritivo, de naturaleza multipropósito (fijación biológica de nitrógeno, aporte de materia orgánica al suelo, disponibilidad de follaje y vainas para consumo animal, sombra al ganado, etc.) y amplio margen de adaptación a climas y suelos (Navas y Restrepo, 2003; García et al., 2006). En el trópico mexicano existe amplia diversidad de estas especies arbóreas (Flores et al., 2006), pero los productores desconocen su potencial como fuente de proteína, cuyo aprovechamiento a través de opciones tecnológicas silvopastoriles pueden intensificar la ganadería regional en armonía con el ambiente agroecológico (Ibrahim et al., 2006).

En la región central del Estado de Chiapas México, algunos productores han observado consumo animal de vainas de algunas arbóreas, así como los efectos tóxicos que producen, como problemas gastroentéricos, abortos e incluso la muerte. Destaca la evaluación del consumo de vainas de quebracho, Acacia farnesiana (Velásquez et al., 2005), como fuente no convencional de proteína disponible para ovinos de lana durante la época seca en esta región; después de $110 \mathrm{~d}$, todos los niveles de inclusión de vainas secas y molidas $(0,10,20$ y $40 \%$, $\mathrm{p} / \mathrm{p})$ produjeron incrementos significativos $(\mathrm{P}>0.05)$ en el peso de los animales, y el análisis beneficio/costo indicó que la dieta con $10 \%$ de vainas fue la más rentable.

De Jesús et al. (2008) efectuaron estudios de palatabilidad de vainas de diferentes leguminosas, donde la cañadonga ( $C$. grandis) no fue de las especies más preferidas, pero destacó por su abundancia de vainas por árbol, peso y tamaño de vainas, propiedades deseables que aseguran su disponibilidad para los animales. En un estudio similar hecho en quebracho en un periodo de 106 $\mathrm{d}$, estos mismos autores reportaron que no hubo diferencia estadística entre la dieta comercial y los niveles de sustitución de ella por cañadonga $(0,5,10,20$ y 40
$\%$ ), además de que no fue tóxica y produjo un efecto reconstituyente en la salud de los ovinos, y la incorporación del $10 \%$ de vainas secas y molidas en la dieta mejoró la relación beneficio/costo de la alimentación de los ovinos.

No obstante, en el Estado de Chiapas la cañadonga es un árbol en peligro de extinción (R. Ordoñez C., 2008 Com. pers. $)^{1}$, y la mayoría de estudios en ella se refieren a su importancia en reforestación, como melífera y a su uso medicinal en casos de disfunción hepática, anemia e infecciones microbianas y fúngicas (Witsberger et al., 1982; Masís et al., 1998); apenas hay unos pocos estudios como planta forrajera y por el uso de hemolizado y pulpa como reconstituyente en la desnutrición de terneros (Montejo et al., 2005; García et al., 2006).

Ante la necesidad de generar información sobre dónde y en qué condiciones se encuentra creciendo la cañadonga en esta región, aquí se estudió el periodo de disponibilidad de vainas, el uso y manejo de los árboles existentes y la capacidad de su producción por árbol, información que será útil para la planeación estratégica de su inclusión en la dieta de rumiantes, particularmente en la época seca en la región. Los objetivos de este estudio fueron conocer la etnobotánica de la cañadonga $(C$. grandis), definir algunas características edafoclimáticas de los sitios donde crece, su morfometría y ciclo fenológico en la región central de Chiapas, así como estimar su rendimiento de vainas por árbol, caracterizar sus vainas y semillas, y definir un modelo de predicción para la producción de vainas.

\section{MATERIALES Y MÉTODOS}

\section{Encuesta, características edafoclimáticas, morfometría y fenología de la cañadonga}

El estudio inició en octubre 2007 y concluyó en 2008. Se aplicó aleatoriamente una encuesta $a d$ hoc a $10 \%$ (201 productores) de los socios de la Unión Ganadera Regional del Estado de Chiapas, para registrar el conocimiento etnobotánico de la especie en la región; es decir, para recabar evidencia sobre su grado de conocimiento y saber cuántos ejemplares tenían en su unidad de producción pecuaria (UPP) y cómo la utilizaban. La encuesta se hizo con un cuestionario con tres preguntas abiertas y cuatro cerradas (Cuadro 1).

\footnotetext{
${ }^{1}$ R Ordoñez C (2008) Propietario de un ejemplar de unos 50 años, quien introdujo la cañadonga a la Región Central del Estado de Chiapas, y ha dado seguimiento a estos árboles en Tapachula y la costa de Chiapas.
} 
Cuadro 1. Cuestionario aplicado a los productores pecuarios de la región central del Estado de Chiapas, sobre el grado de conocimiento de la cañadonga $(C$. grandis).

\begin{tabular}{l}
\hline Nombre del productor (a): \\
Asociación a la que pertenece: \\
Nombre del rancho: \\
Localización __ \\
\hline ¿Conoce el árbol de cañadonga? \\
Sí ( ) \\
Si lo conoce con otro nombre, indique cuál o cuáles: \\
¿Tiene usted este árbol en su rancho? \\
Sí ( ) \\
¿Para qué lo utiliza? \\
¿Sabe que puede utilizarse para la alimentación de los animales? \\
¿En qué especies de animales lo ha utilizado? \\
¿Qué parte de la planta utiliza? \\
$\quad$ Vainas enteras ( ) \\
Vainas molidas ( ) \\
Flores ( ) \\
Otras observaciones: _ Hojas ( )
\end{tabular}

En la región central de Chiapas solamente se pudieron ubicar nueve ejemplares de cañadonga: seis en el municipio de Tuxtla Gutiérrez, dos en áreas urbanas aledañas TXI y TXII, tres en San José Terán (colonia rural) TEI, TEII y TEIII y uno en Plan de Ayala (colonia rural) PA; otros dos árboles en el municipio de San Fernando SFI y SFII; y uno en el municipio de Tecpatán, TP. Previo convenio con los propietarios para desarrollar el presente estudio y recabar datos de su edad, manejo y fertilización, se geoposicionaron mediante un GPSMAP 60CSx Garmín ${ }^{\circledR}$ (Garmin Inc., Taiwan), y se tomaron las medidas morfométricas siguientes: altura, con un clinómetro digital Haglöf ${ }^{\circledR}$ (Haglöf Sweden AB, Langsele, Sweden), diámetro de tallo a la altura del pecho (DAP, a $1.5 \mathrm{~m}$ sobre el suelo) y diámetro de la copa de los árboles. También se tomaron muestras de suelo, a 0 $30 \mathrm{~cm}$ de profundidad, dentro del área de la copa de los árboles, para determinar el pH del suelo. Se identificó la clase de suelo en la que se encontraban, de acuerdo con FAO, única fuente disponible en el Estado de Chiapas.

La caracterización fenológica se efectuó mediante un estudio no destructivo que consistió en dividir en cuatro secciones la copa del árbol, una por punto cardinal, y en cada sección se registraron las observaciones visuales de cuatro personas (el productor y tres integrantes del proyecto), e in situ se determinó la moda del porcentaje estimado para el estado de desarrollo de los componentes morfológicos siguientes: hojas (rebrotes y hojas adultas), flores (yemas, inflorescencias inmaduras y maduras) y vainas denominadas como: a) Comas, después de la pérdida de pétalos cuando sólo quedaba el pistilo en la corona, b) Ejotes, vainas verdes de tamaño similar al de un ejote de frijol; c) Vainas jóvenes, al terminar de crecer a lo largo, para crecer después en grosor; y d) Vainas verdes adultas, al inicio del proceso de maduración. Esto se hizo por periodos semanales en cada árbol y durante un año. La figura 1 ilustra varias etapas fenológicas reproductivas de la cañadonga.

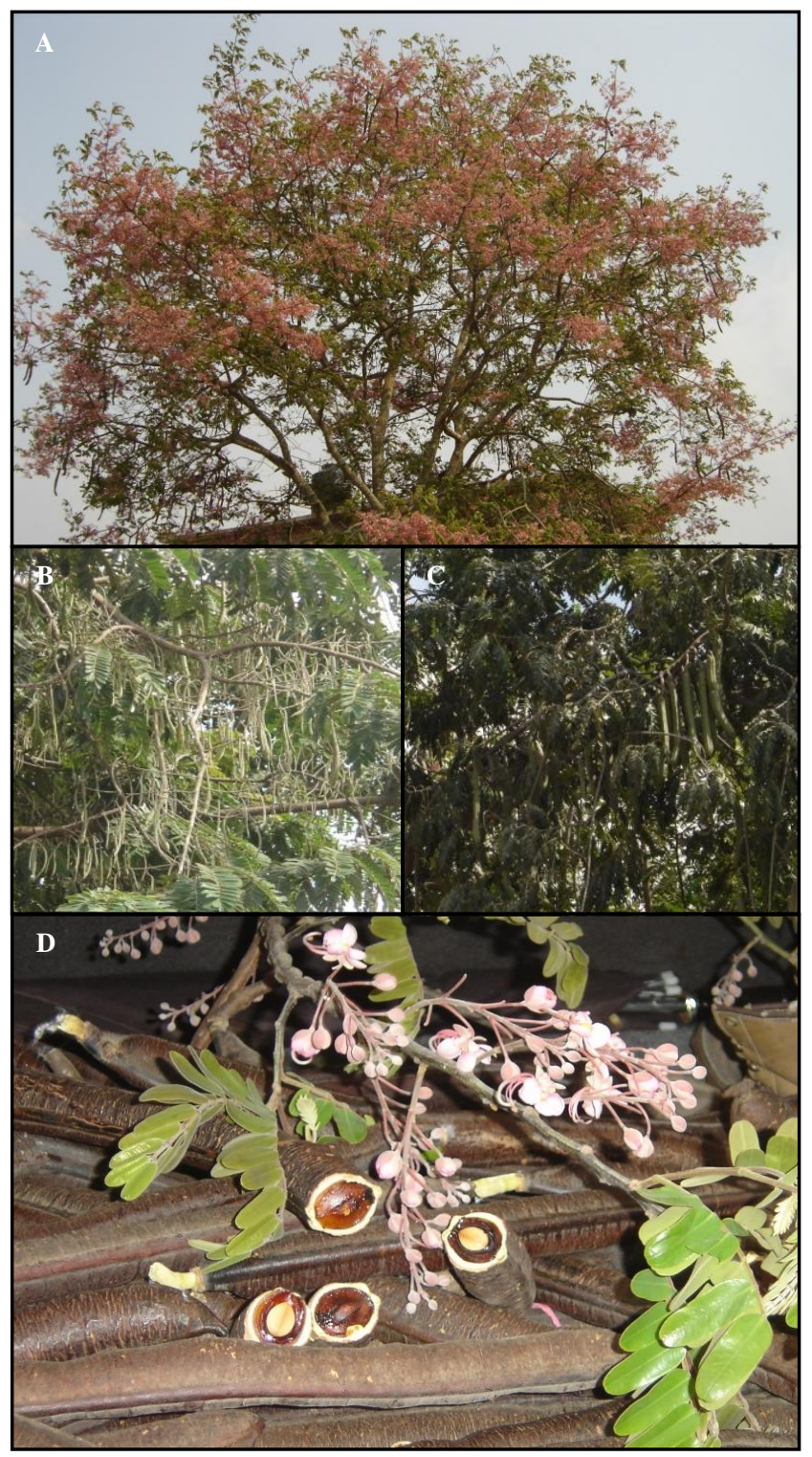

Figura 1. Etapas fenológicas de la cañadonga (Cassia grandis L. f.) en la región central del Edo. de Chiapas. A) Árbol en plena floración; B) Árbol en etapa de vainas jóvenes ("ejotes"); C) Árboles en etapas de vainas adultas verdes; $y$ D) Hojas, flores y vainas maduras.

\section{Rendimiento y caracterización de vainas y semillas}

El rendimiento de vainas maduras por árbol se evaluó al iniciar el periodo de floración en 2008, para no dañar las 
yemas e inflorescencias jóvenes; al momento de la cosecha las vainas adultas y sus semillas estaban completamente formadas y próximas a alcanzar la madurez fisiológica (color parcialmente café). La cosecha se hizo con ayuda de escaleras, pértigas y pinzas de podar; luego se dejaron secar 3 d y se cuantificó el número y peso total de vainas por árbol. A partir de una muestra de $10 \%$ del total de vainas por planta, se evaluaron las siguientes variables: ancho, largo y peso de vainas, número de semillas por vaina, y peso de 100 semillas sanas (cinco lotes por árbol). Los árboles PA y SF II se cosecharon cuando las vainas habían alcanzado la madurez fisiológica (color café oscuro uniforme), debido a que los propietarios no permitieron hacer la cosecha antes. La cañadonga de Tecpatán no formó vainas en 2007 y por ello no pudo incluirse en la evaluación del número y rendimiento de vainas; no obstante, con la disposición de vainas que se formaron en 2006 y las que se habían colectado en 2007 , se incluyó en la evaluación del resto de variables.

\section{Análisis estadístico de la información}

La fenología se presenta de manera descriptiva con las modas de los porcentajes asignados por los escrutadores. Las variables relativas a la evaluación de vainas y semillas, se analizaron mediante un diseño completamente al azar con diferente número de repeticiones, y con la prueba de comparación de medias de Tukey $(\mathrm{P} \leq 0.05)$. En el número y rendimiento (peso total) de vainas por árbol, se calcularon medidas de tendencia central y dispersión. Adicionalmente, se efectuaron análisis de regresión y correlación entre la edad, las variables morfométricas de los árboles y el rendimiento de vainas, conforme a los criterios de Steel y Torrie (1988) y mediante el procedimiento GLM del paquete estadístico SAS Versión 8.2e (SAS Institute, 2003).

\section{Datos climatológicos}

Los datos de precipitación pluvial y temperatura máxima y mínima anuales (únicos factores de clima disponibles) durante 2007 - 2008, se obtuvieron de los registros que la Comisión Nacional del Agua tiene en cada municipio.

\section{RESULTADOS Y DISCUSIÓN}

\section{Etnobotánica, clima, suelo y morfometría de los árboles de cañadonga}

Aunque todos los productores mostraron interés en esta leguminosa, los resultados revelaron que solamente $1 \%$ de los encuestados la conoce y la confunden con la cañafístula (Cassia fistula L.). Los árboles no reciben manejo de poda o fertilización. Ellos utilizan ambas especies como "remedio" para problemas intestinales (lavado) en humanos $(0.05 \%)$ y como carbón o por su aroma fuerte las tiran a la basura $(0.05 \%)$. La información relacionada con su uso medicinal se aproxima a la reportada en la mayoría de países latinoamericanos donde se encuentra esta arbórea, particularmente en El Salvador y Costa Rica, para casos de disfunción hepática, anemia e infecciones microbianas (Masís et al., 1998), pero no se encontraron reportes de que las vainas se utilicen como leña.

Cuadro 2. Geoposición, caracterización y pH del suelo de los sitios donde se ubican los árboles de cañadonga (C. grandis) estudiados en la región central del Edo. de Chiapas. 2007-2008.

\begin{tabular}{|c|c|c|c|c|c|c|c|}
\hline Árbol & $\begin{array}{l}\text { Posición } \\
\text { geográfica }\end{array}$ & $\begin{array}{c}\text { Altitud } \\
(\mathrm{m})\end{array}$ & $\begin{array}{c}\text { Altura del árbol } \\
(\mathrm{m})\end{array}$ & $\begin{array}{c}\text { Diámetro } \dagger \text { tallo } \\
(\mathrm{m})\end{array}$ & $\begin{array}{c}\text { Diámetro de la copa } \\
(\mathrm{m})\end{array}$ & $\begin{array}{c}\text { Edad } \\
\text { (años) }\end{array}$ & $\begin{array}{c}\mathrm{pH} \text { suelo } \\
(1: 2, \text { suelo:agua })\end{array}$ \\
\hline TX I & $\begin{array}{l}\text { LN } 16^{\circ} 44.780^{\prime} \\
\text { LO } 093^{\circ} 07.610^{\prime}\end{array}$ & 589 & 10.35 & 0.245 & 13.0 & 12 & 8.6 \\
\hline TX II & $\begin{array}{l}\text { LN } 16^{\circ} 45.124^{\prime} \\
\text { LO } 093^{\circ} 05.230^{\prime}\end{array}$ & 523 & 13.5 & 0.688 & 18.0 & 20 & 8.2 \\
\hline TE I & $\begin{array}{l}\text { LN } 16^{\circ} 44.942^{\prime} \\
\text { LO } 093^{\circ} 10.622\end{array}$ & 579 & 13.3 & 0.365 & 16.5 & 12 & 8.3 \\
\hline TE II & $\begin{array}{l}\text { LN } 16^{\circ} 44.758^{\prime} \\
\text { LO } 093^{\circ} 10.856^{\prime}\end{array}$ & 588 & 10.4 & 0.245 & 14.5 & 5 & 8.6 \\
\hline TE III & $\begin{array}{l}\text { LN } 16^{\circ} 44.523^{\prime} \\
\text { LO } 093^{\circ} 10.934^{\prime}\end{array}$ & 606 & 8.5 & 0.115 & 7.6 & 3 & 8.2 \\
\hline SF I & $\begin{array}{l}\text { LN } 16^{\circ} 50.153^{\prime} \\
\text { LO } 093^{\circ} 11.573^{\prime}\end{array}$ & 849 & 13.0 & 0.428 & 13.0 & 15 & 7.9 \\
\hline SF II & $\begin{array}{l}\text { LN } 16^{\circ} 49.963, \\
\text { LO } 093^{\circ} 11.585\end{array}$ & 831 & 11.5 & 0.510 & 11.5 & 14 & 8.6 \\
\hline PA & $\begin{array}{l}\text { LN } 16^{\circ} 40.879^{\prime} \\
\text { LO } 093^{\circ} 13.302^{\prime}\end{array}$ & 591 & 17.4 & 0.565 & 13.0 & 13 & 8.0 \\
\hline TP & $\begin{array}{l}\text { LN } 17^{\circ} 07.320^{\prime} \\
\text { LO } 093^{\circ} 18.353^{\prime}\end{array}$ & 530 & 21.0 & 0.405 & 13.4 & 28 & 8.2 \\
\hline
\end{tabular}

A $1.5 \mathrm{~m}$. 
La precipitación registrada en Tecpatán (árbol TP) duplicó la del resto de municipios (2580 vs. $1116 \mathrm{~mm}$ anuales en Tuxtla Gutiérrez, y $1073 \mathrm{~mm}$ en San Fernando). Los ejemplares de cañadonga se encontraron en suelos con diversos grados de alcalinidad y en altitudes por arriba de $800 \mathrm{~m}$, a pesar de que Salazar et al. (2000) reportó que esta especie típicamente crece en suelos ácidos y en altitudes no mayores que la citada (Cuadro 2).

Las dimensiones promedio de los árboles fueron: 12.5 $\mathrm{m}$ de altura, $0.573 \mathrm{~m}$ y $10.4 \mathrm{~m}$ de diámetros de tallo y copa (Cuadro 2). Estos ejemplares resultaron de menor altura que los de El Salvador y otras partes de Centroamérica, donde alcanzan $30 \mathrm{~m}$ (Sandker y Totaro, 2007). No obstante, tanto los valores del tallo como los de altura se ajustan a los reportados para cañadonga y otras especies arbóreas (Sánchez et al., 2004).

\section{Fenología}

La floración de los árboles varió entre sitios. Salvo al inicio de ésta cuando sólo se observaron yemas florales, en todo el periodo coexistieron yemas, inflorescencias inmaduras y maduras. Dado que las flores se presentaron en inflorescencias con órdenes complejos de ramificación, sólo se presenta el porcentaje global de ellas. En general, la floración inició en febrero y terminó en mayo (Figura
2). Conforme transcurrió la floración también ocurrió pérdida de hojas, y en lapsos cortos y ocasionales el árbol solamente tenía flores (una o dos semanas). En el árbol SF I de San Fernando fue notable la presencia de yemas florales desde finales de enero (el ejemplar más precoz); en contraste, el árbol Tuxtla I fue el último en comenzar a florecer y el segundo en concluir esta etapa fenológica. Los árboles alcanzaron la máxima floración entre el 15 y 30 de marzo, salvo los ejemplares TX I, SF II y PA que la presentaron alrededor del 15 de abril. Durante el tiempo que transcurrió la floración, en todos los árboles se estimó una pérdida de flores de 40 a $50 \%$. Según el IRENA (1992), la cañadonga pierde sus hojas en la época seca y cuando el árbol está defoliado inicia la floración, pero esto no se observó en los árboles aquí estudiados, sino que la floración y la defoliación transcurrieron de manera simultánea. El IRENA (1992) también indica que en Costa Rica la floración ocurre de febrero a abril, aunque recientemente se reportó que ocurre de enero a mayo, en tanto que en El Salvador florece de marzo a abril (Salazar et al., 2000). Estos reportes coinciden parcialmente con los resultados aquí obtenidos, donde además se observó polinización anemófila de las flores efectuada por abundantes abejas, como describe PROMABOS (2010).
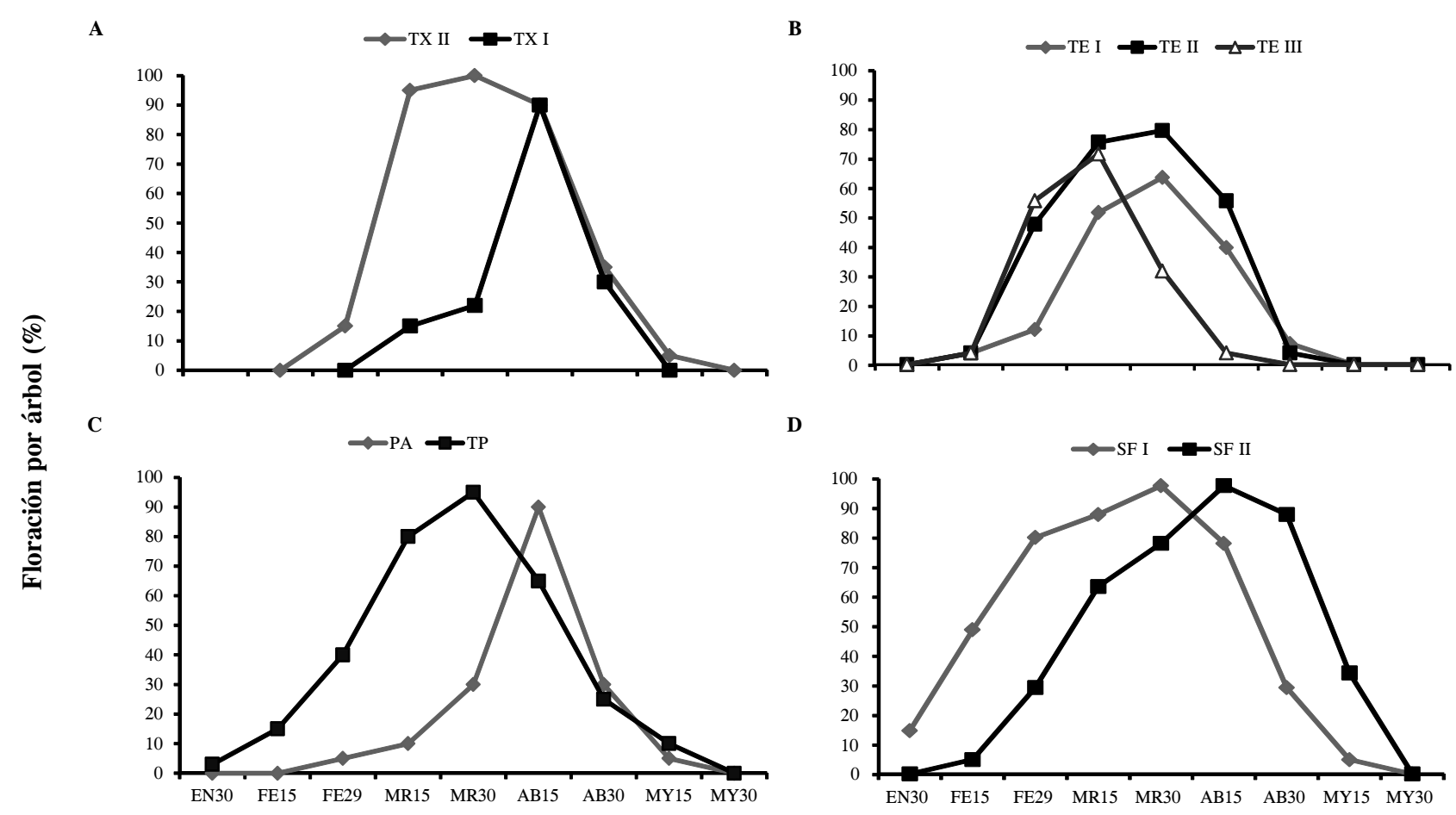

Mes y día

Figura 1. Periodos de floración de árboles de cañadonga (C. grandis) estudiados en la región central del Estado de Chiapas, 2008. TX= Tuxtla Gutiérrez; TE = Terán; $\mathbf{P A}=$ Plan de Ayala; $\mathbf{T P}=$ Tecpatán; $\mathbf{S F}=$ San Fernando. 


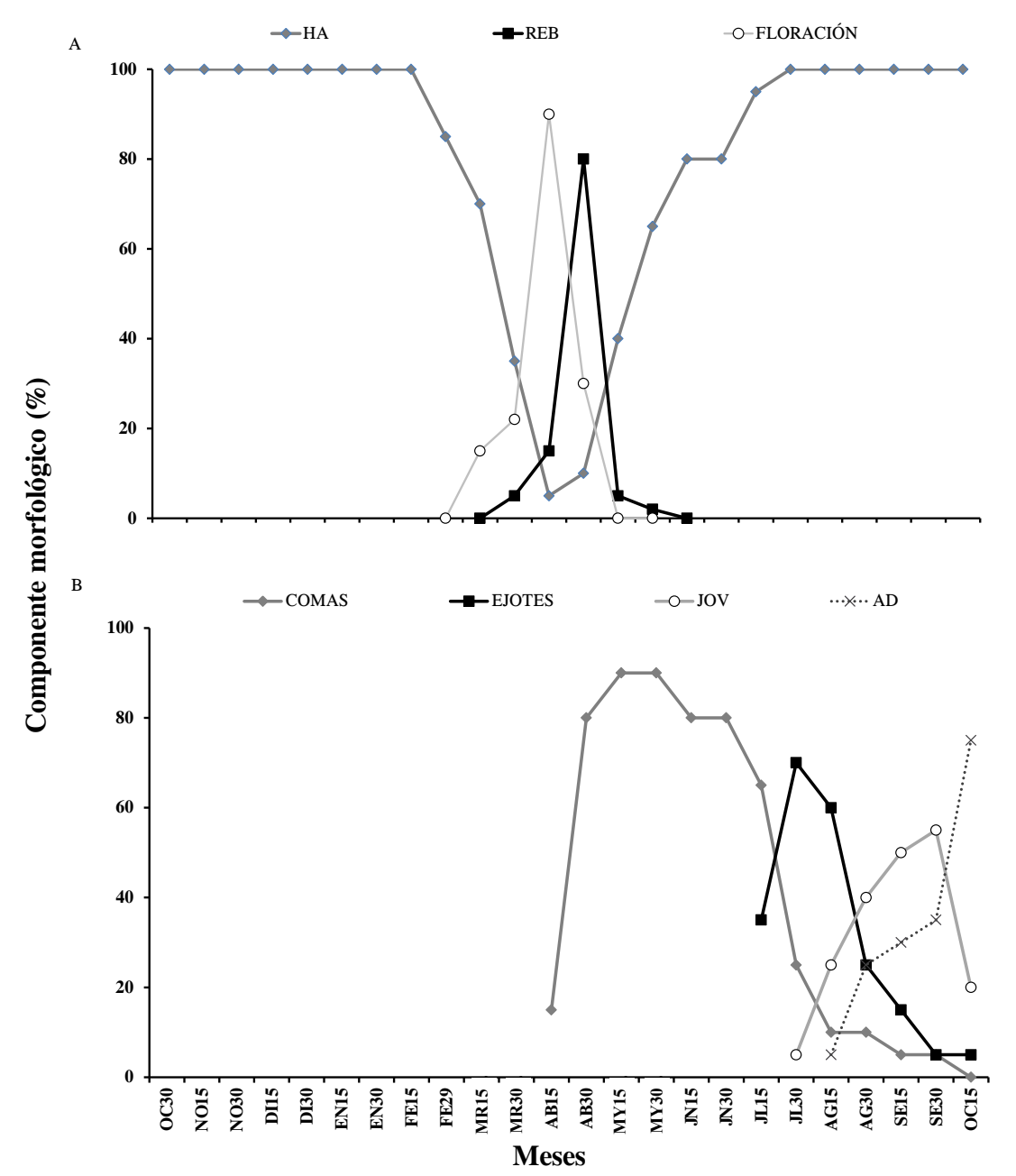

Figura 3. Ciclo fenológico (2008) de la cañadonga (C. grandis, árbol TX I) A) Cinética de hojas, rebrotes, flores $(\mathrm{HA}=$ hojas adultas; $\mathrm{REB}=$ rebrotes; $\mathrm{FLO}=$ floración $) . \mathrm{B})$ Cinética de formación de vainas $(\mathrm{COMAS}=$ pistilos; $\mathrm{EJOTES}=$ similares a ejotes; $\mathrm{JOV}=$ jóvenes; $\mathrm{AD}=$ adultas $)$.

La formación de vainas inició posteriormente a la pérdida de pétalos y sépalos de las flores, cuando solamente quedaron los pistilos adheridos a la corona y comenzaron lentamente a desarrollar las vainas; las esposas de los productores participantes denominan "comas" a estas vainas muy jóvenes y fueron difíciles de observar por el abundante rebrote y crecimiento de las hojas. Después de 8 a 12 semanas alcanzaron el estado que las señoras denominan "ejote", para luego crecer relativamente rápido ( 2 a 4 semanas) y exhibir el estado de vainas jóvenes, las que después de otras dos a tres semanas más alcanzaron el estado de vainas adultas verdes. La cinética de formación de vainas descrita fue muy parecida en todos los ejemplares y por esta razón sólo se presentan datos del ejemplar TX I (Figura 3).

A partir de octubre de 2007, la mayoría de los árboles tenían al menos la mitad de sus vainas adultas verdes; el total de éstas finalizaron la etapa de llenado de grano y llegaron a la madurez fisiológica en aproximadamente 18 semanas (marzo 2008), las vainas en estos ejemplares fueron parcial o totalmente dehiscentes. Las observaciones fenológicas para la cañadonga en Centroamérica (Janzen, 1971; Salazar et al., 2000), coinciden con lo aquí observado, excepto en que los estados de "ejote" y vaina adulta en esa región se alcanzan con un mes de anticipación y las vainas son indeshiscentes.

\section{Características de las vainas, semillas y rendimiento de vainas por árbol}

El peso, largo y ancho de las vainas fueron diferentes $(\mathrm{P}<0.05)$ entre árboles. Los mayores valores $(\mathrm{P}<$ $0.05)$ de peso y largo de vainas se registraron en el ejemplar TP (Cuadro 3), seguido del árbol PA, en tanto que el árbol TE II produjo las vainas más anchas $(\mathrm{P}<$ 0.05). El ancho de las vainas locales es un poco menor 
pero más largas que las reportadas por el IRENA (1992), y menos largas y gruesas que las producidas en Costa Rica donde miden 40 a $65 \mathrm{~cm}$ de largo y 4 a $5 \mathrm{~cm}$ de ancho (Salazar et al., 2000).

La cantidad de semillas por vaina también varió $(\mathrm{P}<$ $0.05)$ entre árboles. Los árboles TX I y SF II presentaron el mayor y menor número total de semillas (81 y 28); la mayor cantidad de semillas sanas (80) se registró en el árbol TX I, y el árbol TX II no produjo semillas vanas (Cuadro 4). Según OFI-CATIE (2008), en Costa Rica forman un promedio de 55 semillas por vaina, que coincide con el promedio aquí obtenido de nueve árboles; en Costa Rica cada kilogramo de semilla de cañadonga tiene 1200 a 3000 semillas, mientras los árboles SF I y TP de Chiapas tenían 728 y 1374 semillas $\mathrm{kg}^{-1}$.

El peso de 100 semillas de los árboles TP y PA superaron $(\mathrm{P}<0.05)$ al resto de los árboles, y las más ligeras correspondieron al árbol SF I. Esto posiblemente se deba a que se cosecharon hasta completar la madurez fisiológica, mientras que en el resto de los árboles, se cosecharon poco antes de su completa madurez. No obstante, el peso de 100 semillas del árbol SF II fue igual al de los ejemplares TX II, TE I y TE II.

El número de vainas por árbol varió de 239 a 1226 (Cuadro 3). El árbol PA formó el mayor número de vainas y produjo el mayor rendimiento $(254 \mathrm{~kg}$ por árbol). Se encontró correlación positiva $\left(\mathrm{r}=0.91^{* *}\right)$ entre la altura del árbol y el rendimiento de vainas (Figura 4). Es de hacer notar que el árbol TE III de 3 años produjo más vainas que el TE II de 5 años, pero las vainas eran de menor tamaño e incluso deformes; un ejemplar de dos años de edad en San José Terán apenas presentó $25 \%$ de flores y 12 vainas. También es importante señalar que los ejemplares TE II y TE III proceden del árbol TE I. Aparentemente, éstos son los primeros datos de estimación de la producción de vainas por árbol de esta leguminosa arbórea.

Cuadro 3. Dimensiones, número y producción de vainas en los árboles de cañadonga ( $C$. grandis) en la región central del Estado de Chiapas, 2008.

\begin{tabular}{|c|c|c|c|c|c|}
\hline \multirow[t]{2}{*}{ Árbol } & \multicolumn{3}{|c|}{ Vainas } & \multirow{2}{*}{$\begin{array}{c}\text { Número de } \\
\text { vainas por árbol }\end{array}$} & \multirow{2}{*}{$\begin{array}{c}\text { Rendimiento } \\
\text { de vainas } \\
\text { (kg por árbol) }\end{array}$} \\
\hline & $\begin{array}{c}\text { Peso } \\
\text { (g) }\end{array}$ & $\begin{array}{l}\text { Largo } \\
\text { (cm) }\end{array}$ & $\begin{array}{c}\text { Ancho } \\
\text { (cm) }\end{array}$ & & \\
\hline TX I & $289.0 \pm 11.1 \mathrm{cde}$ & $43.2 \pm 0.71 \mathrm{~cd}$ & $2.9 \pm 0.05 \mathrm{f}$ & 480 & 110 \\
\hline TX II & $267.7 \pm 115.2$ cde & $33.0 \pm 0.71 \mathrm{f}$ & $3.2 \pm 0.06 \mathrm{def}$ & 913 & 178 \\
\hline TE I & $278.8 \pm 13.7 \mathrm{cde}$ & $36.7 \pm 1.11 \mathrm{def}$ & $3.6 \pm 0.07 \mathrm{ab}$ & 906 & 205 \\
\hline TE II & $313.5 \pm 15.5 \mathrm{cde}$ & $38.8 \pm 0.81 \mathrm{~d}$ & $3.8 \pm 0.06 \mathrm{a}$ & 239 & 51 \\
\hline TE III & $271.3 \pm 10.9 \mathrm{cde}$ & $35.8 \pm 0.65$ ef & $3.5 \pm 0.03 b c$ & 310 & 33 \\
\hline SF I & $203.3 \pm 8.6 \mathrm{f}$ & $33.7 \pm 0.92 \mathrm{f}$ & $3.3 \pm 0.05$ cde & 493 & 111 \\
\hline SF II & $281.0 \pm 10.4 \mathrm{ce}$ & $39.7 \pm 0.74 \mathrm{cde}$ & $3.3 \pm 0.03$ cde & 604 & 78 \\
\hline PA & $379.0 \pm 4.5 \mathrm{~b}$ & $48.6 \pm 0.32 \mathrm{~b}$ & $3.2 \pm 0.02 \mathrm{def}$ & 1226 & 254 \\
\hline $\mathrm{TP}$ & $497.3 \pm 4.3 \mathrm{a}$ & $54.4 \pm 2.69 \mathrm{a}$ & $3.3 \pm 0.05$ cde & ND & ND \\
\hline Promedio & 309.0 & 40.4 & 3.3 & 646 & 127 \\
\hline CV (\%) & 11 & 17 & 7 & 22 & 14 \\
\hline DST & 33.3 & 7.4 & 0.3 & 139 & 18 \\
\hline
\end{tabular}

Medias con letras iguales en cada columna, no son estadísticamente diferentes (Tukey, 0.05 ). ( $\mathrm{g} \pm$ error estándar; $\mathrm{n}=10 \%$ del total de vainas en cada ejemplar). ND = no determinado debido a que el árbol no formó vainas en 2007; CV = coeficiente variación; DST = desviación estándar.

Cuadro 4. Caracterización de semillas en vainas de los árboles de cañadonga (C. grandis) estudiados en la región central del Estado de Chiapas, 2008.

\begin{tabular}{|c|c|c|c|c|}
\hline \multirow{2}{*}{ Árbol } & \multicolumn{3}{|c|}{ Semillas (núm. por vaina) } & \multirow{2}{*}{$\begin{array}{l}\text { Peso de } 100 \text { semillas } \\
(\mathrm{g})\end{array}$} \\
\hline & Total & Sanas & Vanas & \\
\hline TX I & $81 \pm 1.64 \mathrm{a}$ & $80 \pm 1.55 \mathrm{a}$ & $1 \pm 0.33$ ef & $74.0 \mathrm{e}$ \\
\hline TX II & $35 \pm 1.67 \mathrm{~h}$ & $35 \pm 1.67 \mathrm{fg}$ & $0 \pm 0.00 \mathrm{~g}$ & $99.9 \mathrm{c}$ \\
\hline TE I & $70 \pm 3.32 \mathrm{bc}$ & $66 \pm 3.19 \mathrm{~b}$ & $4 \pm 0.79 \mathrm{de}$ & $101.6 \mathrm{c}$ \\
\hline TE II & $72 \pm 2.61 \mathrm{ab}$ & $61 \pm 4.00$ bce & $11 \pm 3.09 \mathrm{bc}$ & $96.3 \mathrm{c}$ \\
\hline TE III & $54 \pm 2.32 \mathrm{defg}$ & $35 \pm 2.07 \mathrm{~g}$ & $19 \pm 1.38 \mathrm{a}$ & $88.1 \mathrm{~d}$ \\
\hline PA & $57 \pm 2.62 \mathrm{def}$ & $53 \pm 0.65$ cde & $4 \pm 0.29 \mathrm{de}$ & $116.6 \mathrm{~b}$ \\
\hline TP & $62 \pm 2.06$ bcde & $59 \pm 2.08$ bcde & $3 \pm 0.05 \mathrm{de}$ & $137.4 \mathrm{a}$ \\
\hline Promedio & $\mathbf{5 7}$ & $\mathbf{5 0}$ & $\overline{7}$ & 98.8 \\
\hline CV (\%) & 17 & 20 & 87 & 20.0 \\
\hline DST & 17 & 17 & 7 & 19.0 \\
\hline
\end{tabular}

Medias con letras iguales en cada columna, no son estadísticamente diferentes (Tukey, 0.05). CV = coeficiente variación; DST = desviación estándar. 


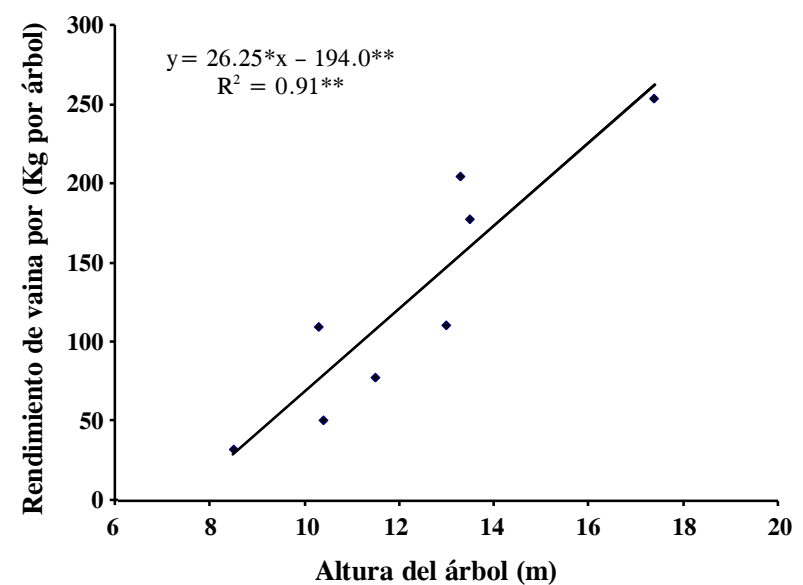

Figura 4. Relación entre la producción de vainas de cañadonga $(C$. grandis) y la altura del árbol, en la región central de Chiapas, 2008. $*$, ** Significativo a $\mathbf{P} \leq 0.05$ y $\mathbf{P} \leq \mathbf{0 . 0 1}$.

Si se considera la producción del árbol más rendidor y que se requieren $14400 \mathrm{~kg}$ de vainas para suplementar un hato de 40 vacas durante $90 \mathrm{~d}$ en la época seca, se requerirían sólo 2 ha ( 25 árboles $\mathrm{ha}^{-1}$ ) para disponer de esa cantidad de vainas. El potencial productivo de esta arbórea es considerablemente alto si se compara con otras especies como el samán (Samanea saman), del cual se requerirían 36 ha para alcanzar la misma cantidad de suplemento (Navas y Restrepo, 2003).

Los resultados acerca de la cantidad de semillas por vaina de estos árboles de Chiapas donde son de naturaleza multipropósito, ofrece la posibilidad de promover su establecimiento en las unidades de producción pecuaria en la región central del estado, como las que hay en Centroamérica, Caribe y norte de Sudamérica (terrenos abiertos, cercos vivos y pastizales), para así ofrecer a la ganadería tropical mayor cantidad de proteína y minerales, que en términos generales se encuentran de manera deficitaria en los pastos (García y Medina, 2006). También sería necesario establecer los posibles factores antinutricios, el valor nutritivo y su repercusión en la aceptabilidad por parte de los rumiantes y otras especies de importancia zootécnica y de la fauna silvestre, para establecer las principales ventajas y limitaciones en el uso de cada especie arbórea disponible en el trópico.

\section{CONCLUSIONES}

La cañadonga fue reconocida solamente por $1 \%$ de los productores de la Unión Ganadera Regional del Estado de Chiapas. En la región la utilizan como medicamento humano ó leña, y no recibe manejo agronómico. Los árboles crecen en diferentes $\mathrm{pH}$ del suelo y altitudes, en comparación con las reportadas en otros países. El periodo máximo de floración varió con la ubicación de los árboles y en general ocurrió de febrero a mayo. La cinética de formación de vainas fue similar en los ejemplares estudiados y la maduración de las vainas fue la etapa más larga del ciclo fenológico de la planta (18 semanas). Hubo diferencias entre árboles en cuanto a las dimensiones y peso de las vainas, así como en las cantidades de semilla total, sana y vana por vaina, y en el peso de 100 semillas. El número de vainas varió de 239 a 1226 por árbol y el rendimiento de 33 a $254 \mathrm{~kg}$ por árbol. La relación entre la altura de los árboles y la producción de vainas fue lineal simple $\left(\mathrm{R}^{2}=0.91 *\right)$, y permite estimar la producción de los árboles.

\section{AGRADECIMIENTOS}

Al Sistema Institucional de Investigación de la Universidad Autónoma de Chiapas (SIINV-UNACH), por el financiamiento de este proyecto en su convocatoria 2007. Al apoyo admirable y desinteresado de Luis López Kolb, estudiante de la Facultad de Medicina Veterinaria y Zootecnia, UNACH.

\section{BIBLIOGRAFÍA}

De Jesús Ch K, R Perezgrovas G, M E Velasco Z, M Salvador F (2008) Ganancia de peso y producción del vellón sucio en corderos suplementados con diferentes niveles de inclusión de "cañadonga" (Cassia grandis). In: Memorias XIV Congreso Nacional de la Asociación Mexicana de Técnicos Especialistas en Ovinocultura A. C. R Grajales Z (ed). 11 y 12 Septiembre de 2008. Tuxtla Gutiérrez, Chiapas, México. pp:1-5.

Enríquez Q J F, F Meléndez N, E E Bolaños A (1999) Tecnología para la Producción y Manejo de Forrajes Tropicales en México. INIFAP, CIRGOC. Campo Experimental Papaloapan. Libro Técnico Núm. 7. Veracruz, México. 262 p.

Flores J S, R M Vermont-Ricalde, J M Kantún-Balam (2006) Leguminosae diversity in the Yucatan Peninsula and its importance for sheep and goat feeding In: Herbivores: Assessment of Intake, Digestibility and the Roles of Secondary Compounds. C A Sandoval-Castro, F D De B Hovell, J F J Torres-Acosta, A Ayala-Burgos (eds). Nottingham University Press. Thrumpton, U K. pp:291-301.

García D E, M G Medina (2006) Composición química, metabolitos secundarios, valor nutritivo y aceptabilidad relativa de diez árboles forrajeros. Zoot. Trop. 24:233-250.

García D E, M G Medina, J Humbría, C Domínguez, A Baldizán, L Cova, M Soca (2006) Composición proximal, niveles de metabolitos secundarios y valor nutritivo del follaje de algunos árboles forrajeros tropicales. Arch. Zoot. 55:377-384.

Ibrahim M, J Rojas, C Villanueva (2006) Tecnologías forrajeras para la intensificación de la ganadería y la conservación de los recursos naturales en el trópico. In: Producción y Manejo de los Recursos Forrajeros Tropicales. M E Velasco Z, A Hernández G, R A Perezgrovas G, B Sánchez M (eds). pp:133-156.

IRENA, Instituto Nicaragüense de Recursos Naturales y del Ambiente (1992) Árboles Forestales Útiles para su Propagación. Instituto Nicaragüense de Recursos Naturales y del Ambiente. Servicio Forestal Nacional, Managua, Nicaragua. $262 \mathrm{p}$.

Janzen D H (1971) Escape of Cassia Grandis L. beans from predators in time and space. Ecology 52:964-979. 
Masís A, A Guadamuz, D Perez, F Chavarría, R Espinoza (1998) Species page de Cassia grandis (Fabaceae). Species Home Pages, Área de Conservación Guanacaste, Costa Rica. Disponible en: http://www.acguanacaste.ac (Marzo 2010).

Mena-Urbina M A, A Hernández-Garay, J F Enríquez-Quiroz, J Pérez-Pérez, J L Zaragoza-Ramírez, M E Velasco-Zebadúa, J Avellaneda-Cevallos (2007) Efecto de asignaciones de forraje, en pastoreo, sobre pasto insurgente y producción de vaquillas en el trópico húmedo. Agrociencia 41:1-12.

Montejo C C E, C M Castañeda S, C O Martínez Y, C F Pérez F, J Duvergel R, W Ramírez S, Y Salgado A (2005) Hemolizado y la cañadonga (Cassia grandis) como reconstituyente en la desnutrición de los terneros. Rev. Veterinaria REDVET 6:1-7. Disponible en: http:www.veterinaria.org/revistas/redvet/n0909 05.html

Navas C A, C Restrepo S (2003) Frutos de leguminosas arbóreas: una alternativa nutricional para ganaderías en el trópico. Agroforestería para la producción animal en América Latina. II. Memorias 2da Conferencia electrónica. FAO. Roma, Italia. pp:283-295. Disponible en: http://www.fao.org/DOCREP/006/ Y4435S/y4435s0o.html

OFI-CATIE, OFI-Centro Agronómico Tropical de Investigación y Enseñanza (2008) Leguminosae Caesalpinioide. Cassia grandis L.f. Árboles de Centroamérica. Un Manual Para Extensionistas. J Cordero, D H Boshier (eds). Centro Agronómico Tropical de Investigación y Enseñanza. Turrialba, Costa Rica. 1080:439- 442.
PROMABOS, Proyecto de Manejo de Abejas y del Bosque (2010) Carao. Árboles melíferos para reforestar. Disponible en: www.bio.uu.nl/promabos/arbolesmeliferos/pdf files/Carao (Marzo 2010).

Salazar R, C, Soihet, J M Méndez (2000) Manejo de Semillas de 100 Especies Forestales de América Latina. Volumen 1. Serie Técnica Manual No. 41, CATIE, Turrialba, Costa Rica. 204 p.

Sánchez A J, F A Pacariote, S Alfonzo, R Flores (2004) Arquitectura y fenología de las especies Prosopis juliflora y Acacia tortuosa en el semiárido del estado de Falcón, Venezuela. Instituto Nacional de Investigaciones Agrícola, Falcón, Venezuela. Arch. Latinaom. Prod. Anim. 12 (Supl. 1):72-81.

Sandker M, L Totaro (2007) Árboles melíferos para reforestar. Disponible en: www.bio.uu.nl/promabos/arbolesmeliferos/ pdf_files/Carao.pdf. (Octubre 2009).

SAS Institute Inc. (2003) The Analyst Application. 2nd ed. Cary, NC, USA. $500 \mathrm{p}$.

Steel R G D, J H Torrie (1988) Bioestadistica: Principios y Procedimientos. McGraw-Hill. México. $622 \mathrm{p}$

Velásquez A J, R Perezgrovas G, M E Velasco Z, L Zaragoza M, G Rodríguez G (2005) Evaluación de vainas de quebracho (Acacia farnesiana) en alimentación de ganado lanar. Arch. Zoot. 54:535-540

Witsberger D, D Current, E Archer (1982) Árboles del Parque Deininger. Dirección de Publicaciones del Ministerio de Educación. San Salvador, El Salvador. 336 p. 